\title{
EVIDENCE FOR A STRONGLY COUPLED DIPOLE MODE WITH INSUFFICIENT DAMPING IN TTF FIRST ACCELERATING MODULE
}

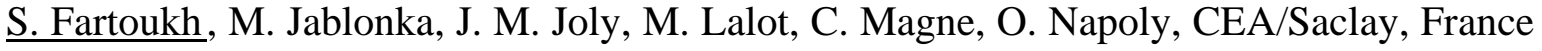 \\ M. Bernard, LAL/Orsay, France, \\ N. Baboi, S. Schreiber, S. Simrock, H. Weise, DESY/Hamburg, Germany
}

\begin{abstract}
A beam experiment has been conducted on the first accelerating module of the TESLA Test Facility (TTF) to investigate transverse higher order modes (HOM) in the superconducting cavities. By injecting the beam with a transverse offset and by modulating the intensity of the $216 \mathrm{MHz}$ bunch train with a tunable frequency in the 0 $108 \mathrm{MHz}$ range, transverse HOMs can be excited resonantly. On a resonance the outgoing bunch orbits, measured with a broadband BPM, are transversely modulated at the same frequency. A dipole mode at about $2585 \mathrm{MHz}$, belonging to the third passband of the TESLA cavities, has been excited and observed in this way, with unexpected low damping in 2 out of the 8 cavities of the module.
\end{abstract}

\section{AIM OF THE EXPERIMENT}

The transport of very low emittance beams along the TESLA linac requires transverse higher order mode damping at a level defined in [1]. HOM couplers mounted on the beam tubes of the TESLA 9-cell superconducting cavities, have been designed to achieve damping with $\mathrm{Q}$ factors of the order of $10^{4}$ to $10^{5}$, depending on the mode coupling impedance, especially for the first two dipole passbands. Modes from higher passbands, supposedly above cutoff, are expected to propagate and therefore to couple efficiently to the HOM couplers except for few so-called "trapped" modes whose energy is concentrated in the central cells of the cavity. Of the experiments proposed at the TTF to verify with beam that the HOM damping is adequate and to detect unwanted trapped modes, the one reported here, described in [2], consists of excitation of higher order modes with the sidebands of the bunch train $f_{b}=216 \mathrm{MHz}$ harmonics generated by modulation of the bunch charge with a variable frequency $F$ in the range of 0 to $108 \mathrm{MHz}$. If the beam is injected off axis in the accelerating module, these sidebands build up in the Fourier transform of beam dipole moment and a resonant transverse instability can develop when one of the sidebands and one transverse HOM frequency coincide, i.e. $n f_{b} \pm F=f_{\text {HOM }}$. Hence, at the cavity exit, the transverse position of the beam is modulated at the frequency $F$ with in phase and out of phase components.
A broadband BPM can be used to detect these oscillations.

\section{EXPERIMENTAL SETUP}

The first accelerating module of TTF includes eight 9cell superconducting cavities at $1.3 \mathrm{GHz}$ RF frequency. HOM couplers are located on both sides of each cavity to extract the power lost by the beam at high current. During the experiment, the thermoionic gun delivered up to $8 \mathrm{~mA}$ of beam current at a pulse duration of $600 \mu \mathrm{s}$ with bunch frequency of $216.7 \mathrm{MHz}$. The additional experimental set-up includes the following devices:

- A tuneable voltage modulator at the cathode of the gun, providing the required charge modulation along the bunch train, with a modulation amplitude nearly up to $100 \%$ of the nominal $37 \mathrm{pC}$ bunch charge.

- A 'dog-leg' magnet (half a chicane) in front of the accelerating module providing a steady horizontal offset, in the range from 0 to $20 \mathrm{~mm}$.

- A broadband cavity BPM [3] to measure individual positions of the bunches located about $10 \mathrm{~m}$ downstream of the accelerating module exit.

Entering the module, beam energy was about $9 \mathrm{MeV}$. The gradients of the eight cavities in the module were set to a minimum value of $2 \mathrm{MV} / \mathrm{m}$ in order to maximise the sensitivity of the beam to HOM deflections. With such energy settings and quadrupole magnets off, the BPM is at a focal point for parallel trajectories entering the module. This makes it easier to measure HOM kicks in the cavities.

\section{OBSERVATION OF THE $2585 \mathrm{MHz}$ HOM}

As discussed in [2], all HOMs can be excited by sidebands of the beam harmonics, at frequencies $n f_{b} \pm F$, by scanning the charge modulation frequency $F$ through half a Brillouin zone $\left[0, f_{b} / 2\right]$. Dipole modes are in principle excited by injecting the beam off-axis in the cavities. By doing so, a beam instability was observed on the broadband BPM for $F=15.022 \mathrm{MHz}$ with a short beam pulse of $35 \mu$ s and $5 \mathrm{~mA}$ current, and a large injection offset of about $20 \mathrm{~mm}$. The beam pulse length could be increased to up to $500 \mu$ s while remaining on the HOM resonance. Fig. 1 shows the comparison of the BPM envelope signals with charge modulation off or on for a $400 \mu$ s long beam pulse. With modulation off, the 
broadband BPM integrates a high but constant $100 \mathrm{mV}$ level of beam induced noise, although the beam is steered through its centre. This is due to the rejection of the sum signal into the difference signal BPM antennas. While the modulation is on, the beam offset reaches a maximum of about $10 \mathrm{~mm}$ but, due to the excessive noise no quantitative measurement could be made. A damped oscillatory behaviour of the envelope signal is visible in Fig. 1 with a period of about $125 \mu$ s, on top of the constant envelope of the beam oscillations reached at the steady state. This behaviour was later explained, and reproduced by simulations, by the fact that the modulation frequency was off HOM-resonance by about $8 \mathrm{kHz}(1 / 125 \mu \mathrm{s})$. The resonant oscillations then reach a steady state after some damped overshooting. Simulations predict that for a perfectly on-resonance excitation, the oscillations reach the aperture limit within the $35 \mu$ s short pulse: this agrees with the observed veto from the beam loss protection system occurring at every attempt to fine tune the modulation frequency towards lower values of $F$.

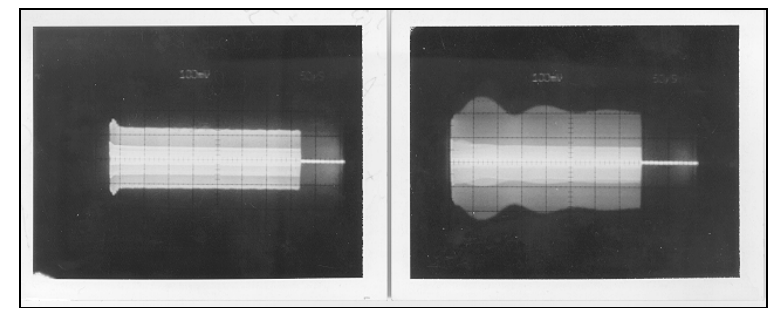

Figure 1: BPM signal on oscilloscope for $400 \mu \mathrm{s}$ beam pulse without (left) and with (right) charge modulation

\section{HOM RF CHARACTERISATION}

The beam experiment revealed the existence of a HOM with a frequency $f_{\text {ном }}=n f_{b} \pm(15.022 \mathrm{MHz}-8 \mathrm{kHz})$ in the accelerating module. While detuning cavities \#1 and \#2 had no effect on the beam instability, detuning cavity \#3 by about $\pm 16 \mathrm{kHz}$ in the fundamental mode did suppress the instability. It was then possible to measure and identify the mode at the HOM coupler outputs of cavity \#3 (cavity $\mathrm{S} 10$ in Table 1) with a spectrum analyser. The frequency domain signal around the $12^{\text {th }}$ beam harmonics at $2.6 \mathrm{GHz}$ in Fig.2 shows a beam modulation side-band peak enhanced by the HOM resonance on the low side of this harmonics. The same signal, when analysed in time domain in Fig.3, shows the $35 \mu$ s beam-pulse cavity loading, followed by the relaxation of the field amplitude with a characteristic time $\tau=110 \mu \mathrm{s}$. The dipolar nature of the mode was demonstrated by checking the perfect linear dependance of the height of the amplitude maximum with the offset of the beam entering the module, over a range from 0 to $20 \mathrm{~mm}$.
The resonant modes of cavity \#3 were studied over a wider range of frequencies while the beam was turned off. As shown by Fig.4, the measured HOM is the highest frequency mode of the $3^{\text {rd }}$ dipole passband. Its frequency is:

$$
f_{\text {ном }}=2584.986 \mathrm{MHz} \pm 1 \mathrm{kHz} .
$$

The damping factor is then given by :

$$
Q=\frac{2 \pi f_{\mathrm{HOM}} W}{P_{d}}=\frac{2 \pi f_{\mathrm{HOM}} \tau}{2} \approx 9 \times 10^{5}
$$

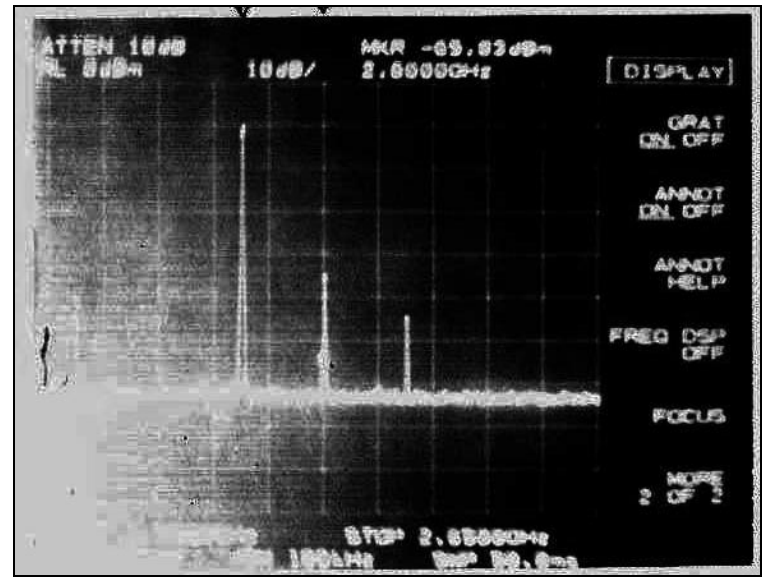

Fig.2 Sidebands around the $2.6 \mathrm{GHz}$ beam harmonics

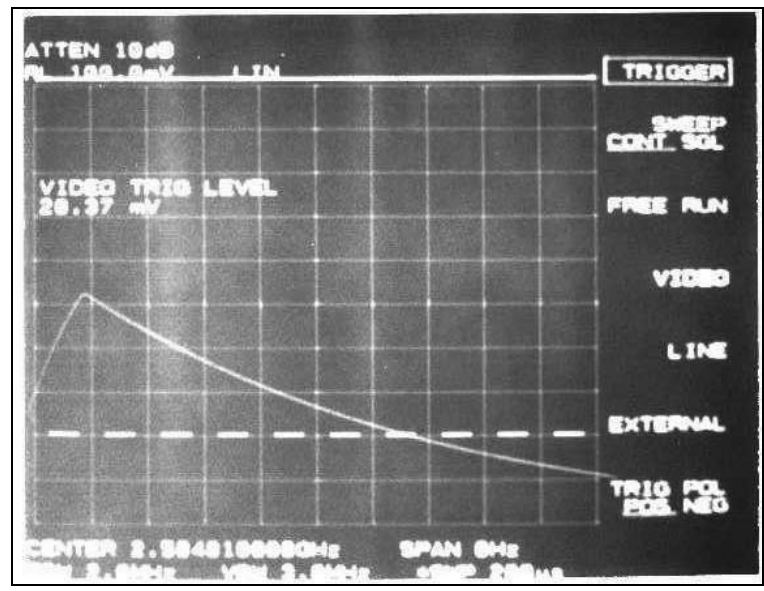

Fig.3 Time domain signal from HOM pickup on the $2585 \mathrm{MHz}$ mode

An URMEL [4] calculation of the $3^{\text {rd }}$ passband of the TESLA cavity was performed in parallel [5] showing that the highest frequency mode of this passband has indeed a large beam coupling impedance

$$
\frac{1}{a^{2}}\left(\frac{R}{Q}\right) \approx 15 \Omega / \mathrm{cm}^{2} .
$$

It is foreseen to compare this prediction with a quantitative measurement of the beam displacement 
using a BPM with an improved resolution in a future experiment.

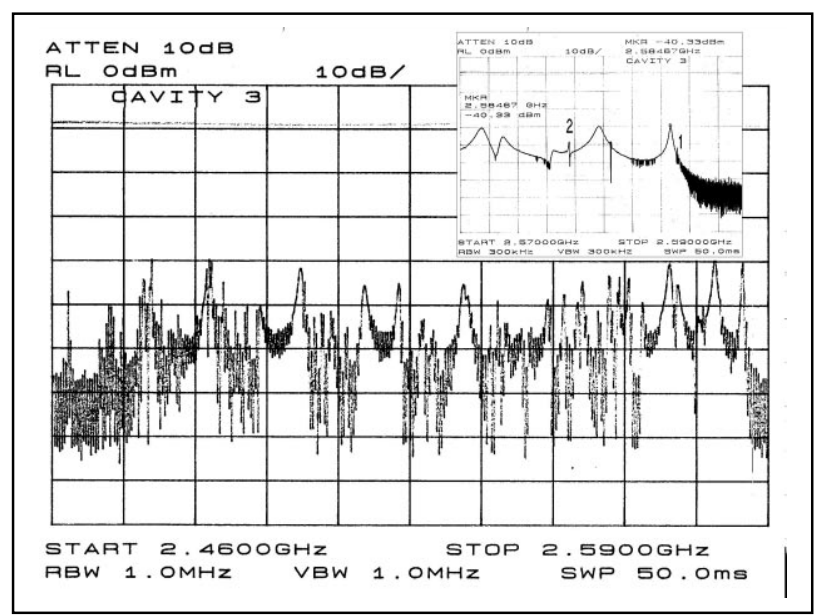

Fig.4: Third dipole passband of cavity \#3. The high frequency edge of this passband is zoomed, showing the $2585 \mathrm{MHz}$ (1) and $2577.5 \mathrm{MHz}$ (2) very sharp modes discussed in the text.

\section{OTHER MEASURED MODES}

Three other modes have been excited and observed with the intensity modulated beam. The HOM excitation signal, taken either from the HOM coupler outputs or from the BPM itself, was analysed with a spectrum analyser, like in the previous section.

- $\quad 2586 \mathrm{MHz}$ mode in cavity \#6: a close inspection of the last mode of third dipole passband of the eight cavities (cf. Table 1) showed, in cavity \#6 (S11), the same excitation curve as the one shown in Fig.3 with a time decay constant of $\tau=8 \mu \mathrm{s}$. It corresponds to a quality factor $Q=6.5 \times 10^{4}$. No measurable excitation could be observed in other cavities.

- $2577.5 \mathrm{MHz}$ mode in cavity \#3: this mode also belongs to the $3^{\text {rd }}$ dipole passband, as can be seen in Fig.4. Its coupling impedance [5] is also large, about $9 \Omega / \mathrm{cm}^{2}$. Its excitation curve gave $\tau=22 \mu$ s which corresponds to $Q=1.8 \times 10^{5}$.

- $1876 \mathrm{MHz}$ mode in cavity \#3: this well-known TM110 mode of the first dipole passband has a high coupling impedance of about $9 \Omega / \mathrm{cm}^{2}$. In cavity \#3, and for one polarisation, it appears to be less efficiently damped than in the other cavities [6] with a measured $\tau=20 \mu \mathrm{s}$, corresponding to $Q=1.2 \times 10^{5}$. This damping is at the limit of the TESLA tolerances [1].
Table 1: Cavities of TTF Module \#1

\begin{tabular}{|l|c|c|}
\hline Name & $\begin{array}{c}\text { HOM coupler } \\
\text { type }\end{array}$ & $\mathbf{3}^{\text {rd }}$ dipole passband \\
\hline D3 & fixed & $2458-2548 \mathrm{MHz}$ \\
\hline S8 & dismountable & $2458-2576 \mathrm{MHz}$ \\
\hline S10 & dismountable & $2471-2585 \mathrm{MHz}$ \\
\hline D1 & fixed & $2464-2562 \mathrm{MHz}$ \\
\hline D2 & fixed & $2462-2560 \mathrm{MHz}$ \\
\hline S11 & dismountable & $2480-2586 \mathrm{MHz}$ \\
\hline D4 & fixed & $2456-2556 \mathrm{MHz}$ \\
\hline S7 & dismountable & $2472-2576 \mathrm{MHz}$ \\
\hline
\end{tabular}

\section{CONCLUSION}

A higher order $2585 \mathrm{MHz}$ dipole mode has been observed and excited with beam with low damping, $Q=$ $9 \times 10^{5}$ in cavity $\# 3$ and $Q=6.5 \times 10^{4}$ in cavity \#6, in two out of eight cavities of the first TTF cryomodule. Identifying this mode with the highest mode of the third dipole passband leads to quite a high transverse coupling parameter $1 / a^{2}\left(R / Q_{\perp}\right) \cong 15 \Omega / \mathrm{cm}^{2}$. This is notably above the TESLA tolerance for the preservation of the vertical emittance in the linac. The origin of the insufficient damping of this mode and of its non-systematic character is not yet understood. A localised HOM coupler defect is of course one possibility. The large cavity-to-cavity spread in HOM frequencies, with highest and similar frequencies in cavities \#3 and \#6, may also be a clue. This observation is currently being completed by RF and beam measurements at the cold module. On the other hand, in order to complete the search of dangerous HOMs, it is foreseen to equip the new TTF photo-injector with a pockell-cell device which allows to modulate the charge of a $54 \mathrm{MHz}$ bunch train.

\section{REFERENCES}

[1] "TESLA Conceptual Design Report”, R. Brinkmann, G.Materlick, J.Rossbach and A. Wagner Editors, DESY 1997-048, (1997)

[2] "A New Method to Detect the High Impedance Dipole Modes of TESLA Cavities", S. Fartoukh, CEA/Saclay preprint, DAPNIA/SEA-98$18,(1998)$

[3] "High Resolution BPM for Future Colliders", C. Magne, M. Juillard, M. Lalot, A. Mosnier, B. Phung, Y. Lussignol and R. Bossart, LINAC98 Conf., Chicago, (1998)

[4] "On the computation of resonant modes in cylindrically symmetric cavities", T. Weiland, NIM 216 (1983).

[5] We thank S. Chel for doing this calculation while the experiment was going on.

[6] G. Kreps, unpublished. 